21

\title{
Светоиндуцированные решетки, создаваемые с помощью пары коротких терагерцовых импульсов, не перекрывающихся в резонансной среде
}

\author{
(C) Р.М. Архипов ${ }^{1,2}$, А.В. Пахомов ${ }^{2}$, М.В. Архипов ${ }^{1,2}$, \\ И. Бабушкин ${ }^{3}$, Н.Н. Розанов ${ }^{2,4,5}$ \\ ${ }^{1}$ Санкт-Петербургский государственный университет, \\ 199034 Санкт-Петербург, Россия \\ 2 Университет ИТМО, \\ 197101 Санкт-Петербург, Россия \\ ${ }^{3}$ Institute of Quantum Optics, Leibniz University Hannover, \\ 30167 Hannover, Germany \\ ${ }^{4}$ Государственный оптический институт им. С.И. Вавилова, \\ 199053 Санкт-Петербург, Россия \\ ${ }^{5}$ ФТИ им. А.Ф. Иофффе, \\ 194021 Санкт-Петербург, Россия \\ e-mail: arkhipovrostislav@gmail.com
}

Поступила в Редакцию 16.06.2018 г.

Показана возможность создания светоиндуцированных решеток разности заселенностей с помощью пары коротких терагерцовых импульсов для управления терагерцовым излучением. Импульсы когерентно взаимодействуют со средой и не перекрываются в среде. Исследованный эффект открывает новые возможности в терагерцовой спектроскопии с помощью коротких импульсов.

DOI: $10.21883 /$ OS.2018.10.46713.166-18

\section{Введение}

В последнее время вызывает значительный интерес генерация коротких импульсов в терагерцовом (ТГц) диапазоне $(0.1-30 \mathrm{THz})[1-5]$. ТГц излучение имеет огромное количество различных применений в биологии и медицине [6,7], беспроводных линиях связи [8] и т.д. На сегодняшний день удается получить ТГц импульсы с длительностью порядка периода колебаний электромагнитной волны в ТГц диапазоне [1-5]. В терагерцовом диапазоне лежат колебательные и вращательные переходы в различных молекулах, а также частоты некоторых элементарных возбуждений в полупроводниковых материалах и диэлектриках $[3,9,10]$. Поэтому короткие импульсы ТГц излучения активно применяются в спектроскопии $[3,9,10]$.

Когда длительность входного импульса меньше времени релаксации и поляризации $T_{2}$, разности заселенностей $T_{1}$ среды, возможно возникновение когерентного взаимодействия короткого импульса со средой. При этом возникают осцилляции Раби атомной инверсии и поляризации [11]. Возможно также создание светоиндуцированных решеток поляризации и разности заселенностей в резонансной среде в условиях, когда импульсы не перекрываются в среде [12-21]. Такой подход к формированию решеток отличается от традиционного, в котором светоиндуцированные решетки создаются при интерференции квазимонохроматических пучков, перекрывающихся в среде [22]. Дифракция света на таких решетках находит различные применения в нелинейной оптике и спектроскопии, например, для измерения оптических характеристик среды, см. монографию [22] и цитируемую в ней литературу.

Возможность создания решеток с помощью пары неперекрывающихся импульсов при когерентном взаимодействии связана с тем, что первый импульс оставляет после себя осциллирующую на частоте резонансного перехода поляризацию среды, что проявляется в наличии волны поляризации, бегущей в направлении распространении импульса. Эта волна существует в течение времени порядка $T_{2}$. Если на интевале времени короче $T_{2}$ в среду войдет второй импульс, распространяющийся в противоположном направлении, то в результате его взаимодействия с бегущей волной поляризации в среде может возникнуть решетка разности заселенностей [12-21].

Возможность создания решеток с помощью последовательности неперекрывающихся в среде импульсов была изучена в оптическом диапазоне теоретически и экспериментально в случае длинных имульсов [12-16], а также теоретически в случае одноцикловых и субцикловых аттосекундных в оптическом диапазоне частот [17-21]. В [23,24] изучалась динамика светоиндуцированных структур при столкновении аттосекундных оптических импульсов в резонансной среде. Однако создание решеток в оптической области с помощью аттосекундных одноцикловых и субцикловых импульсов требует больших значений амплитуд электрического 
поля ( 10 $\left.10^{4}-10^{5} \mathrm{ESU}\right)$, что делает процесс наведения решеток трудным с экспериментальной точки зрения.

С учетом упомянутого выше интереса к генерации ТГц импульсов, в настоящей работе рассматривается возможность создания решеток разности населенностей с помощью пары коротких терагерцовых импульсов. При этом частота резонанса среды также лежит в терагерцовой области. Использование терагерцовых импульсов, как мы увидим далее, значительно упрощает процесс наведения решеток на практике, так как позволяет использовать ТГц импульсы малой амплитуды, на порядки меньшей, чем в случае аттосекундных импульсов. Более того, в случае колебательных переходов, частоты которых лежат в ТГц области, дипольный момент перехода системы типа гармонический осциллятор обратно пропорционален частоте перехода [25]. Поэтому в ТГц области можно ожидать большие значения дипольных моментов (в десятки Дебай), что также позволяет использовать импульсы малой амплитуды для получения больших значений частот Раби. А это также упрощает наблюдение когерентных эффектов в виде осцилляций Раби и создание решеток в терагерцовой области по сравнению со случаем, когда для формирования решеток используются мощные аттосекундные импульсы. Обсуждается возможность применения данных решеток в спектроскопии колебательных переходов молекул с помощью коротких ТГц импульсов.

\section{Теоретическая модель и полученные результаты}

Рассмотрим резонансную среду с частотой колебательного перехода в терагерцовой области области. Для простоты будем описывать среду в двухуровневом приближении. Взаимодействие двухуровневой среды с последовательностью коротких терагерцовых импульсов $E(t)$ описывается с помощью системы уравнений Блоха, определяющих эволюцию недиагонального элемента матрицы плотности $\rho_{12}$ и разности заселенностей (инверсии) $n=\rho_{11}-\rho_{22}$ между основным и возбужденным состоянием вещества. Данная система уравнений имеет вид $[25,26]$

$$
\begin{gathered}
\frac{\partial \rho_{12}(t)}{\partial t}=-\frac{\rho_{12}(t)}{T_{2}}+i \omega_{0} \rho_{12}(t)-\frac{i}{\hbar} d_{12} E(t) n(t), \\
\frac{\partial n(t)}{\partial t}=-\frac{n-1}{T_{1}}+\frac{4}{\hbar} d_{12} E(t) \operatorname{Im} \rho_{12}(t) .
\end{gathered}
$$

Параметры модели: $d_{12}$ - дипольный момент резонасного перехода, $\omega_{0}$ - частота перехода, $\hbar-$ приведенная постоянная Планка. Длительность импульсов и рассматриваемых интервалов времени предполагается меньше времен релаксации $T_{1}$ и $T_{2}$, поэтому сначала мы пренебрежем релаксационными членами. Электрическое поле терагерцовых импульсов берется в виде

$$
\begin{aligned}
E(t) & =E_{0} \exp \left(\frac{-t^{2}}{\tau_{p}^{2}}\right) \sin \omega_{0} t \\
& +E_{0} \exp \left(\frac{-(t-\tau)^{2}}{\tau_{p}^{2}}\right) \sin \left(\omega_{0}[t-\tau]\right) .
\end{aligned}
$$

Здесь $\tau$ - задержка между импульсами.

Система уравнений (1)-(2) в пренебрежении релаксационными членами имеет наглядное решение, описывающее наведение решеток разности населенностей, в предельном случае длинных импульсов, длительность которых много больше периода резонансного перехода среды [21]. В таком случае электрическое поле можно представить в виде произведения медленно меняющейся огибающей $\tilde{E}(t)$ и быстро осциллирующего множителя:

$$
E(t)=\tilde{E}(t) \sin \omega_{0} t
$$

После введения частоты Раби

$$
\Omega(t)=\frac{d_{12} \tilde{E}(t)}{\hbar}
$$

в рассматриваемом предельном случае $\Omega(t) \ll \omega_{0}$ система уравнений $(1),(2)$ может быть приведена к интегрируемому виду путем усреднения по периоду резонансного перехода среды [21]. Тогда, как было показано в [21], инверсия населенности после первого импульса переводится в ноль, $n(t, \tau)=0$, под действием импульса с площадью

$$
\int_{-\infty}^{+\infty} \Omega(t) d t=\frac{\pi}{2} .
$$

Затем второй импульс такой же площади (4), приходящий с временной задержкой $\tau$, создает в среде периодическое распределение (решетку) разности населенностей [21]:

$$
n(\tau)=-\cos \omega_{0} \tau
$$

Для демонстрации возникновения решеток в ТГц диапазоне и проверки аналитического выражения (5) мы воспользуемся той же процедурой, которая использовалась ранее для анализа возникновения решеток в оптическом диапазоне с помощью аттосекундных импульсов $[17,20,21]$. Система уравнений (1),(2) с полем (3) интегрировалась численно на временном интервале, много большем длительности импульсов. Указанная процедура проводилась для каждого значения задержки между импульсами $\tau$ из выбранного интервала. В случае протяженной среды, частицы которой распределены вдоль оси $z$, введенная задержка между импульсами $\tau$ может быть интерпритирована как момент времени $z / c$ прихода импульса в точку среды, имеющую координату $z$. Значение инверсии после окончания действия импульсов запоминалось. Выбранное значение частоты 
резонанса среды $\omega_{0}=6.28 \cdot 10^{12} \mathrm{rad} / \mathrm{s}(1 \mathrm{THz})$ соответствует периоду собственных колебаний $T_{0}=\frac{2 \pi}{\omega_{0}}=1 \mathrm{ps}$, дипольный момент перехода $d_{12}=20 \mathrm{D}$. Амплитуда импульса возбуждения $E_{0}=23.5 \mathrm{ESU}(\sim 7000 \mathrm{~V} / \mathrm{cm})$, что вполне реализуемо экспериментально [1-4]. Временами релаксации пренебрегалось. Длительность импульса $\tau_{p}=2 \mathrm{ps,}$ что также легко достичь экспериментально. Несущая частота импульса совпадает с частотой резонанса среды $\omega_{0}$. Амплитуда импульса выбиралась таким образом, чтобы инверсия принимала нулевое значение после окончания действия первого импульса. Отметим, что длительность импульса больше периода колебаний резонансного перехода среды, поэтому двухуровневое приближение оправдано. Кроме того, результаты экспериментов показывают, что осцилляции Раби возможны также в сложных многоуровневых системах, типа полупроводников [27]. Результаты эксперимента [27] хорошо согласуются с результами моделирования с использованием двухуровневой модели, несмотря на то, что импульсы возбуждения имели широкий спектр и длительность порядка периода колебаний световой волны. Наконец, результаты расчетов, приведенные в работе [21], показали возможность существования решеток разности населенностей в четырехуровневой схеме в оптическом диапазоне. Поэтому в настоящей работе мы будем использовать двухуровневое приближение.

Рисунок $1, a$ иллюстрирует зависимость инверсии $n(\tau)$ после окончания действия импульсов, полученную в результате численных расчетов и построенную с помощью формулы (5). Видно, что результат расчета по аналитической формуле (5) прекрасно согласуется с результатами численного расчета с помощью системы уравнений (1)-(3). Двумерная диаграмма рис. $1, b$ показывает временную зависимость инверсии под действием поля двух ТГц импульсов (3) для каждого значения задержки между импульсами. Из рис. 1 видно, что инверсия имеет периодическую зависимость от задержки, что в случае протяженной среды, расположенной вдоль оси $z$ (когда $\tau=z / c$, где $c-$ скорость света), соответствует возникновению гармонической решетки разности заселенностей под действием поля двух ТГц импульсов.

Учтем теперь конечность времен релаксации среды. Рисунок 2 иллюстрирует ту же самую зависимость, что и на рис. 1 , но при $T_{1}=1 \mathrm{~ns}, T_{2}=5 \mathrm{ps}$. Очевидно, что конечные времена релаксации приведут к затуханию решетки разности заселенностей и уменьшению амплитуды инверсии с ростом задержки между импульсами (рис. 2). Данное обстоятельство может быть использовано для измерения времени релаксации $T_{2}$ (дефазировки колебательных переходов) с помощью дифракции пробного поля на индуцированной решетке разности населенностей, что было ранее продемонстрировано экспериментально в оптическом дипазоне при использовании длинных импульсов [13-15].
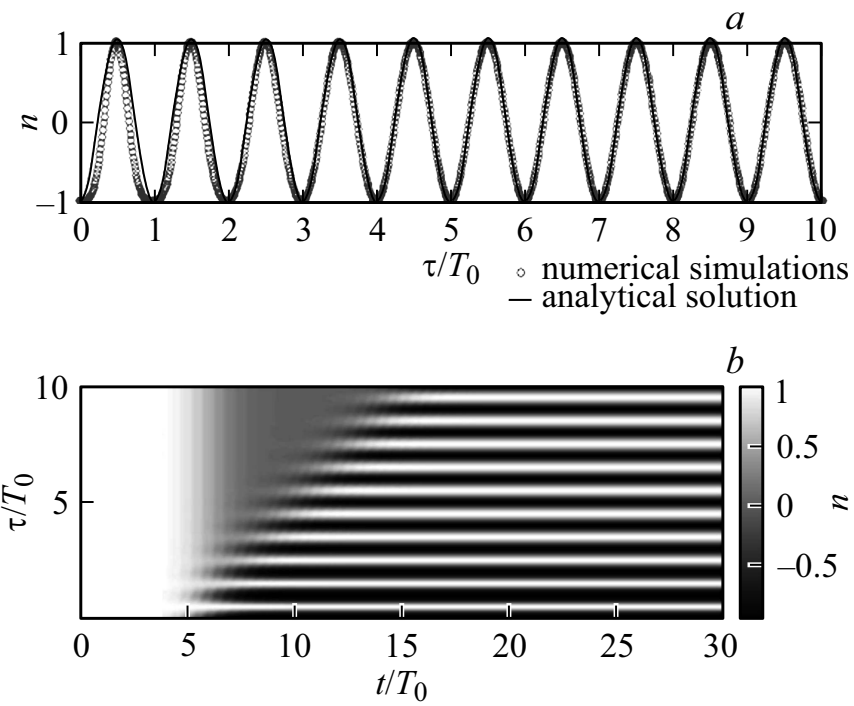

Рис. 1. (a) Зависимость разности заселенностей после окончания действия импульсов от задержки между импульсами $n(\tau)$, полученная в результате численного расчета (точки), аналитическая зависимость $n(\tau)$, построенная по формуле (5) (сплошная линия). (b) Временная зависимость разности заселенностей от значения задержки $\tau$ между двумя импульсами, полученная с помощью численного расчета. $T_{1}=T_{2}=\infty$.
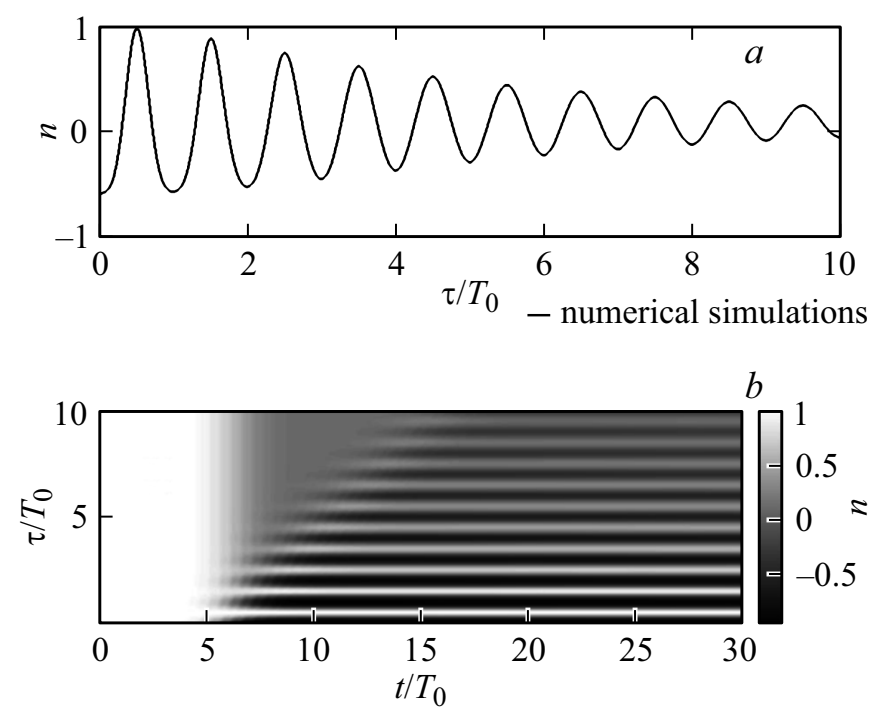

Рис. 2. (a) Зависимость разности заселенностей после окончания действия импульсов от задержки между импульсами $n(\tau)$, полученная в результате численного расчета. $(b)$ Временная зависимость разности заселенностей от значения задержки $\tau$ между двумя импульсами, полученная с помощью численного расчета. $T_{1}=1 \mathrm{~ns}, T_{2}=5 \mathrm{ps}$.

\section{Заключение}

Таким образом, в настоящей работе продемонстрирована возможность создания решетки разности заселенностей в ТГц диапазоне частот с помощью пары коротких ТГц импульсов, не перекрывающихся в среде. Большие 
дипольные моменты переходов в ТГц колебательных переходах позволяют использовать для создания решеток ТГц импульсы гораздо меньшей амплитуды по сравнению с изученным ранее случаем создания решеток в оптическом диапазоне с помощью аттосекундных импульсов. Показано, что конечные времена релаксации приводят к затуханию решетки со временем.

Изученный эффект может применяться в ТГц спектроскопии колебательных переходов в различных молекулах с помощью коротких ТГц имульсов. Например, дифракция пробного импульса малой амплитуды на индуцированной описанной выше решетке разности населенностей может быть использована для измерения времени дефазировки молекулярных колебаний $T_{2}$.

В заключение отметим, что исследованный эффект также возможно ожидать в полупроводниковых структурах, используемых в ТГц квантово-каскадных лазерах, которые активно изучаются в настоящее время [28-31]. Возможность наблюдения решеток в данных структурах усиливается тем, что в работе [32] была экспериментально продемонстрирована возможность осцилляций Раби в квантово-каскадных лазерах.

Исследованные в работе решетки могут быть также использованы для управления направлением распространения трехмерных диссипативных солитонов [33]. Работа частично поддержана грантом РНФ 18-12-00075.

\section{Список литературы}

[1] Reiman K. // Rep. Progr. Phys. 2007. V. 70. P. 1597.

[2] Roskos H.G., Thomson M.D., Kress M., Loeffler T. // Laser Photon. Rev. 2007. V. 1. P. 349.

[3] Lepeshov S., Gorodetsky A., Krasnok A., Rafailov E., Belov P.// Laser Phot. Rev. 2016. V. 11. P. 1600199.

[4] Babushkin I., Kuehn W., Köhler C., Skupin S., Berge L., Reimann K., Woerner M., Herrmann J., Elsaesser T. // Phys. Rev. Lett. 2010. V. 105. P. 053903.

[5] Babushkin I., Skupin S., Husakou A., Kóhler C., CabreraGranado E., Berge L., Herrmann J. // Phys. Rev. Lett. 2010. V. 105. P. 053903.

[6] Murakami H., Toyota Y., Nishi T., Nashima S. // Chem. Phys. Lett. 2012. V. 519. P. 105.

[7] Wu X., Quan B., Pan X., Xu X., Lu X., Gu C., Wang L. // Biosens. Bioelectron. 2012. V. 42. P. 626.

[8] Akyildiz I.F., Jornet J.M., Han C.// Phys. Commun. 2014. V. 12. P. 16.

[9] Parrott E.P., Zeitler J.A. // Appl. Spec. 2015. V. 69. P. 1.

[10] Jepsen P.U., Cooke D.G., Koch M. // Laser Photonics Reviews. 2011. V. 5. P. 124.

[11] Аллен Л., Эберли Дж. Оптический резонанс и двухуровневые атомы. М.: Мир, 1978; Allen L., Eberly J. H. Optical resonance and two-level atoms. N. Y.: Wiley, 1975.

[12] Abella I.D., Kurnit N.A., Hartmann S.R. // Phys. Rev. 1966. V. 141. P. 391.

[13] Штырков Е.И., Лобков В.С., Ярмухаметов Н.Г. // Письма в ЖЭТФ. 1978. Т. 27. № 12. С. 685; Shtyrkov E.I., Lobkov V.S., Yarmukhametov N.G. // JETP Lett. 1978. V. 27. N 12. P. 648.
[14] Shtyrkov E.I., Nevelskaya N.L., Lobkov V.S., Yyarmukhametov N.G. // Phys. Stat. Sol. (b). 1980. V. 98. P. 473.

[15] Szczurek M., Kuśnierz M. // Opt. Commun. 1989. V. 74. P. 121.

[16] Штырков Е.И. // Опт. и спектр. 2013. Т. 114. № 1. С. 105.; Shtyrkov E. I. // Opt. Spectrosc. 2013. V. 114. N 1. C. 96.

[17] Архипов Р.М., Архипов М.В., Бабушкин И., Розанов Н.Н. // Опт. и спектр. 2016. Т. 121. № 5. С. 810; Arkhipov R.M., Arkhipov M.V., Babushkin I., Rosanov N.N. // Opt. Spectrosc. 2016. V. 121. N 5. P. 758.

[18] Arkhipov R.M., Arkhipov M.V., Babushkin I.V., Demircan A., Morgner U., Rosanov N.N. // Opt. Lett. 2016. V. 41. P. 4983.

[19] Архипов Р.М., Архипов М.В., Пахомов А.В., Бабушкин И., Розанов Н.Н. // Квант. электрон. 2017. Т. 47. № 7. С. 589; Arkhipov R.M., Arkhipov M.V., Babushkin I., Pakhomov A.V., Rosanov N.N. // Quantum Electronics. 2017. V. 47. N 7. P. 589.

[20] Arkhipov R.M., Arkhipov M.V., Pakhomov A.V., Babushkin I., Rosanov N.N. // Las. Phys. Lett. 2017. V. 14. N 9. P. 1.

[21] Arkhipov R.M., Pakhomov A.V., Arkhipov M.V., Babushkin I., Demircan A., Morgner U., Rosanov N.N. // Sci. Rep. 2017. V. 7. N 1. P. 12467.

[22] Eichler H.J., Günter E., Pohl D.W. Laser-Induced Dynamic Gratings. Berlin, Heidelberg, N. Y., Tokyo: Springer-Verlag, 1981.

[23] Архипов Р.М., Архипов М.В., Пахомов А.В., Бабушкин И., Розанов Н.Н. // Опт. и спектр. 2017. V. 123. № 4. С. 600; Arkhipov R.M., Arkhipov M.V, Pakhomov A.V., Babushkin I., Rosanov N.N. // Opt. Spectrosc. 2017. V. 123. N 4. P. 610.

[24] Архипов Р.М., Архипов М.В., Пахомов А.В., Жигулева Д.О., Розанов Н.Н. // Опт. и Спектр. 2018. V. 124. N 4. C. 510. Arkhipov R.M., Arkhipov M.V, Pakhomov A.V., Zhiguleva D.I., Rosanov N.N. // Opt. Spectrosc. 2018. V. 124. N 4. P. 541.

[25] Ахманов С.А., Никитин С.Ю. Физическая оптика. М.: Наука, 2004; Akhmanov S.A., Nikitin S.Y. Physical optics. Clarendon Press, 1997.

[26] Yariv A. Quantum electronics. (John WieLy \& Sons, N. Y., 1988).

[27] Mücke O.D., Tritschler T., Wegener M., Morgner U., Kärtner F.X. // Phys. Rev. Lett. 2001. V. 87. N 5. P. 057401.

[28] Wang F. et al. // Optica. 2015. V. 2. P. 944.

[29] Barbieri S., Ravaro M., Gellie P. et al. // Nat. Photon. 2011. V. 5. P. 306.

[30] Bachmann D., Rösch M., Süess M.J., Beck M., Unterrainer K., Darmo J., Faist J., Scalari G. // Optica. 2016. V. 3. P. 1087

[31] Tzenov P., Babushkin I., Arkhipov R., Arkhipov M., Rosanov N., Morgner U., Jirauschek C. // New Journal of Physics. 2018. V. 20. N 5. P. 053055.

[32] Choi H., Gkortsas V., Diehl L., Bour D., Corzine S., Zhu J., Hofler G. et al. // Nature Photonics. 2010. V. 4. N 10. P. 706.

[33] Veretenov N.A., Fedorov S.V., Rosanov N.N. // Phys. Rev. Lett. 2017. V. 119. P. 263901. 